\title{
Extracting Multidimensional Phase Space Topology from Periodic Orbits
}

\author{
Stephan Gekle, ${ }^{1}$ Jörg Main, ${ }^{1}$ Thomas Bartsch, ${ }^{2}$ and T. Uzer ${ }^{2}$ \\ ${ }^{1}$ Institut für Theoretische Physik 1, Universität Stuttgart, 70550 Stuttgart, Germany \\ ${ }^{2}$ Center for Nonlinear Science, Georgia Institute of Technology, Atlanta, Georgia 30332-0430, USA
}

(Dated: February 26, 2018)

\begin{abstract}
We establish a hierarchical ordering of periodic orbits in a strongly coupled multidimensional Hamiltonian system. Phase space structures can be reconstructed quantitatively from the knowledge of periodic orbits alone. We illustrate our findings for the hydrogen atom in crossed electric and magnetic fields.
\end{abstract}

PACS numbers: 05.45.-a, 05.45.Jn, 45.20.Jj, 32.60.+i

Periodic motion is widely recognized as the most prominent feature in a wide range of dynamical systems, from astronomy [1, 2, 3], molecular vibrations [4, 5], chemical reactions [6], particle accelerators [], atomic and molecular physics [8, 9, 10 to fluid dynamics, e.g., statistics of turbulent flow 11]. Because periodic orbits are the fundamental building blocks of the dynamics, they offer a road map to the intricate geometrical and dynamical structure in a multidimensional phase space. Unfortunately, this map is hard to decipher: Highdimensional systems usually possess enormous numbers of periodic orbits whose geometric appearance in configuration space gives no useful hint at their systematics [9, 10]. Indeed, it is not even clear a priori that a systematic organization of periodic orbits should exist at all.

The hydrogen atom in crossed electric and magnetic fields is typical in this respect. It serves as a paradigm of strongly coupled multidimensional systems because it can be investigated both experimentally and theoretically $[9,10,12$. However, after two decades of intense scrutiny, the overall phase space structure still defies a complete

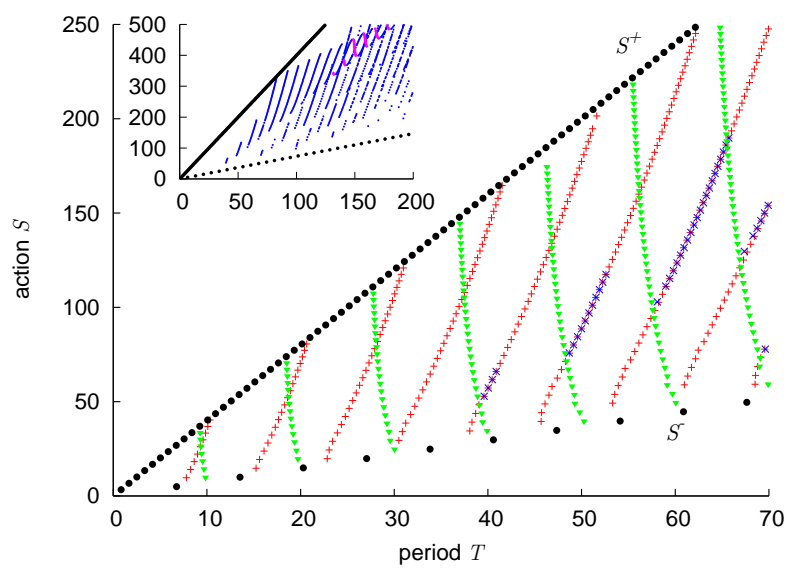

FIG. 1: (color online). Periodic orbits at $E=-1.4$ a.u., $F=0.5$ a.u. FPOs and their repetitions are shown with black circles, $T_{2}^{\mathrm{p}}$ POs with red plus symbols, $T_{2}^{\mathrm{n}}$ with green triangles, $T_{3}^{\mathrm{p}}$ with blue crosses, and $T_{3}^{\mathrm{n}}$ with magenta diamonds. The inset presents the 3 -torus POs in an enlarged $T-S$ range. understanding. In particular, no pertinent symbolic dynamics are known. Although extensive lists of periodic orbits (POs) are available in the literature [9, 10, 13, 14], no comprehensive ordering scheme for POs in three degrees of freedom has been proposed so far, nor has it been shown how POs can provide insight into higherdimensional phase space structures. That such a scheme should exist becomes clear from Fig. 1 We find that the periods and actions of most periodic orbits (POs) in the crossed-fields hydrogen atom fall into clearly discernible series. It is the central aim of this paper to elucidate the dynamical origin of this remarkable structure. We will show that a hierarchical ordering of periodic orbits arises from the hierarchy of invariant tori in an integrable limiting case, and we will develop the tools needed to characterize this organization in a non-integrable system beyond two degrees of freedom. In the course of this work, the nomenclature used in Fig. 1 will be made clear.

The Hamiltonian for the electronic motion in crossedfields hydrogen reads, in atomic units,

$$
H=\frac{1}{2} \mathbf{p}^{2}-\frac{1}{r}+\frac{B}{2}\left(p_{y} x-p_{x} y\right)+\frac{B^{2}}{8}\left(x^{2}+y^{2}\right)-F x .
$$

Here $\mathbf{r}=(x, y, z)$ are the usual Cartesian coordinates, $\mathbf{p}=\left(p_{x}, p_{y}, p_{z}\right)$ the conjugate momenta, and $r=$ $\sqrt{x^{2}+y^{2}+z^{2}}$. The magnetic field $B$ points in the $z$ direction and the electric field $F$ in the $x$ direction. Exploiting the scaling property of the classical Hamiltonian (1), we can set $B=1$ a.u. without loss of generality. We take $F=0.5$ a.u. and consider two energies, $E=-1.5$ a.u. and $E=-1.4$ a.u., slightly below and slightly above the classical ionization threshold $E_{\mathrm{I}}=-2 \sqrt{F}$. To identify POs, we employ a simple shooting algorithm, which like any other numerical PO search is not guaranteed to find all POs. However, our method of torus construction does not require the knowledge of the complete set of POs.

To illustrate the mechanism that leads to the hierarchical ordering of POs, we will first study a two-dimensional subsystem of the crossed-fields hydrogen atom, namely the $x$-y-plane perpendicular to the magnetic field. Its dynamics can conveniently be discussed with the help of a Poincaré surface of section such as Fig. 2. The plot shows two elliptic (stable) fundamental periodic orbits (FPO), 


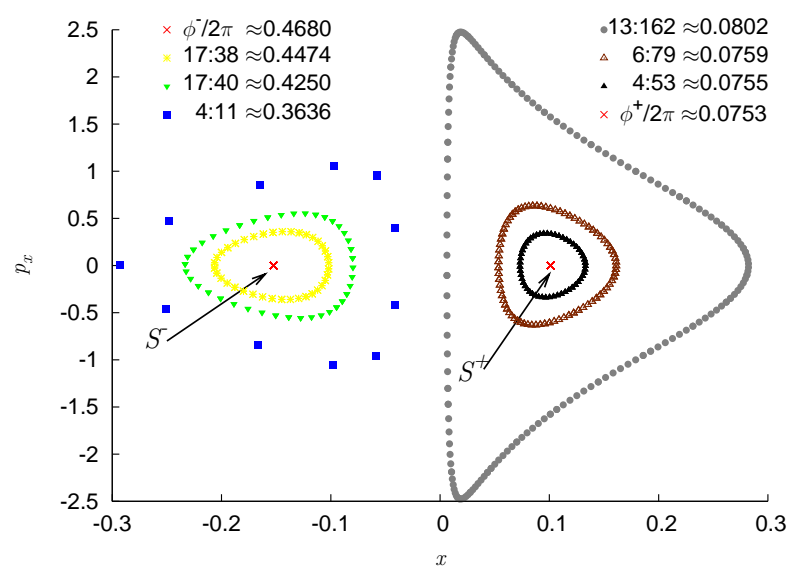

FIG. 2: (color online). Periodic orbits in the Poincaré surface of section $y=0$ for the planar subsystem, $E=-1.5$ a.u., $F=$ 0.5 a.u. Non-fundamental POs are labeled by their winding ratios.

called $S^{+}$and $S^{-}$, surrounded by POs of larger periods. The latter arise in stable/unstable pairs according to the Poincaré-Birkhoff theorem [15]. (For graphical reasons, only one orbit of each pair is displayed in Fig. 2) They can be thought of as being generated from the breakup of a resonant invariant torus in an integrable limiting case of the dynamics. In the situation of Fig. 2] we say that the FPOs serve as the organizing centers for the longer orbits that surround them.

Similar structures can be expected to exist in higher dimensions and have indeed been observed in integrable systems [5]. Given that in a Hamiltonian system of $L$ degrees of freedom there can be invariant tori of dimension up to $L$, one might expect to find an entire hierarchy: FPOs serve as organizing centers for families of POs that arise from the breakup of 2-dimensional tori (2-torus POs), which in turn organize families that are generated from 3-dimensional tori (3-torus POs), and so on up to the maximum dimension. However, Poincaré surface of section plots are hard to visualize beyond two degrees of freedom. Therefore, they do not provide a practical tool to identify these families of POs and to diagnose their mutual relationships. We will here introduce a quantitative method to accomplish these two tasks in a multidimensional system and we will demonstrate in a 3 -dimensional example that the hierarchy outlined above does indeed exist. The hierarchy of POs that we derive mirrors the hierarchy and topology of their originating invariant tori in the integrable limit, which is also the hierarchy of surviving invariant Kolmogorov-Arnold-Moser (KAM) tori [15] of the full non-integrable dynamics.

The key conceptual tools that we use to elucidate this hierarchy are action variables and winding numbers. In the integrable limit, any motion is confined to an invariant torus 15. that is characterized by a set of conserved action variables $\mathbf{I}$. Their conjugate angles $\boldsymbol{\theta}$ determine

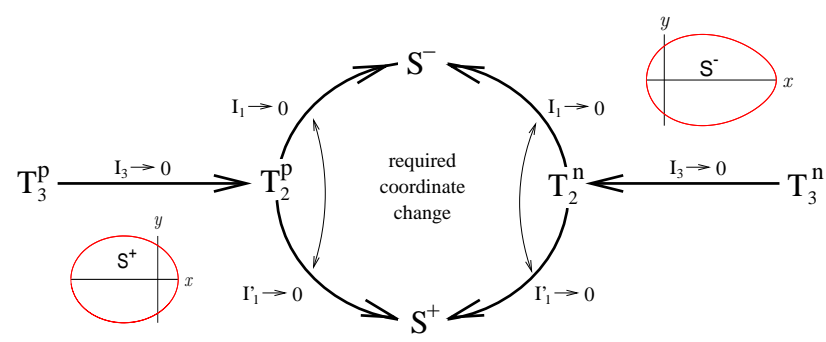

FIG. 3: (color online). The hierarchy of $n$-torus POs in the crossed-fields hydrogen atom at $E=-1.5$ a.u., $F=0.5$ a.u. Insets show the FPOs in configuration space.

the position on the torus. If an $n$-torus carries POs, integer winding number $w_{1}, \ldots, w_{n}$ can be assigned to it so that the angle $\theta_{i}$ runs from 0 to $2 \pi \cdot w_{i}$ before the orbit closes. Because winding numbers are topological properties, they remain meaningful in the non-integrable case, where the resonant tori have broken up into isolated POs. Using the winding numbers $\mathbf{w}$, the total action can be written as $S=\mathbf{w} \cdot \mathbf{I}(\mathbf{w})$, where $\mathbf{I}(\mathbf{w})$ are the action coordinates of the originating torus. In fact, the actions depend only on the ratios of winding numbers, e.g., $w_{1} / w_{2}$ and $w_{3} / w_{2}$ for 3 -torus POs or $w_{1} / w_{2}$ for 2-torus POs.

While the assignment of winding numbers to a given PO is simple with the help of a Poincaré surface of section plot, it is in itself a difficult problem in more than two degrees of freedom. The geometric appearance of the orbits in configuration space does not provide a useful guide. A major part of this Letter will therefore be devoted to the development of a viable method to assign winding numbers to individual POs.

Once winding numbers (and the associated action variables, the calculation of which is then straightforward) are available, the situation in Fig. 2 can be characterized by the following quantitative criteria: (i) The stability angles $\phi_{1}^{ \pm}$of the FPOs $S^{ \pm}$(i.e. the phase angles of the unimodular eigenvalues of their stability matrices) describe the rotation that each FPO imposes upon its neighborhood. The winding ratios of the 2 -torus POs converge toward $\phi_{1}^{ \pm} / 2 \pi$ as the FPOs are approached. (ii) In the same limit, the action variable corresponding to the degree of freedom along the FPO converges to the action of the FPO, and (iii) the action variable for the motion transverse to the FPO, which is given by the area the (original) invariant torus encloses in the Poincaré plane, tends to zero. We will use these three criteria to identify analogous situations in higher dimensions, and we will show that in a similar manner families of 2-torus POs can themselves serve as organizing centers for families of 3 -torus POs. Notice that although the choice of action-angle coordinates and the associated winding numbers is not unique, within the hierarchical structure the organizing center imposes a specific coordinate system (along/transverse to the FPO) upon the 
family it organizes.

POs that are remnants of the same torus have nearly identical periods and actions, thus each point in Fig. 1 represents POs generated from a single torus. As in the two-dimensional case, 2-torus POs occur in doublets. By contrast, 3-torus POs arise in groups of four [16]. At the basis of the hierarchy of POs in the crossed-fields hydrogen atom at $E=-1.5$ a.u. there are the FPOs (or 1-torus POs) $S^{+}$and $S^{-}$that were described in 13,14$]$. They are shaped nearly like Keplerian ellipses in the invariant $x$-y-plane. 2-torus and 3-torus POs are arranged in series with positive or negative slopes in the $T$-S $S$-diagram of Fig. 1 which form the $T_{2,3}^{\mathrm{p}}$ and $T_{2,3}^{\mathrm{n}}$ families, respectively. The POs contained in the $x-y$ plane and illustrated in Fig. 2 form the family $T_{2}^{\mathrm{p}}$. The series of 2-torus POs end at boundary lines marked by the FPOs and their repetitions. This observation suggests that each of the FPOs serves as an organizing center for both families $T_{2}^{\mathrm{p}}$ and $T_{2}^{\mathrm{n}}$. We will show below that this is indeed the case. (Notice that in three degrees of freedom each FPO has two transverse degrees of freedom characterized by two different stability angles. It can therefore organize two different families of 2-torus POs.) In addition, we will see that the families $T_{2}^{\mathrm{p}, \mathrm{n}}$ themselves act as organizing centers for the families $T_{3}^{\mathrm{p}, \mathrm{n}}$ of 3 -torus POs. The entire hierarchy thus obtained is illustrated schematically in Fig. 3. At the energy $E=-1.4$ a.u., above the ionization saddle point, we find the same general structure, except that the FPO $S^{-}$is surrounded by a region of ionizing trajectories and thus cannot serve as an organizing center for the $T_{2}^{\mathrm{p}, \mathrm{n}}$.

Winding numbers for the 2-torus POs $T_{2}^{\mathrm{p}}$ and $T_{2}^{\mathrm{n}}$ can be assigned as follows: Counting the series in Fig. 1yields the first winding number $w_{1}$. The second winding number $w_{2}$ is found by counting the POs within one series from bottom to top. It remains only to determine with what values the counting is to start in both cases. To this end, we use Fourier expansions of the coordinate functions $x(t)$ and $z(t)$. These spectra show two major peaks. One of them agrees with the series number $w_{1}$ (starting with $w_{1}=1$ in the leftmost series), whereas the location of the second is identified with the second winding number $w_{2}$ (starting with $w_{2}=2$ for the shortest orbit in the leftmost series). The Fourier expansions thus confirm the validity of the simple counting scheme described above.

For the 2-torus POs we obtain the action coordinates I shown in Fig. 4 together with the actions and stability angles $\phi_{1,2}$ of the FPOs $S^{ \pm}$. The limiting values for high winding ratios of the $T_{2}^{\mathrm{p}}$ and $T_{2}^{\mathrm{n}}$ families coincide with $\phi_{1}^{-} / 2 \pi$ and $\phi_{2}^{-} / 2 \pi$ of $S^{-}$, respectively. As the maximum winding ratio is approached, $I_{2}$ converges towards the action of the FPO, whereas $I_{1}$ vanishes. According to the three criteria listed above, we can therefore conclude that the FPO $S^{-}$serves as an organizing center for the two families $T_{2}^{\mathrm{p}}$ and $T_{2}^{\mathrm{n}}$ of 2-torus POs, and we can

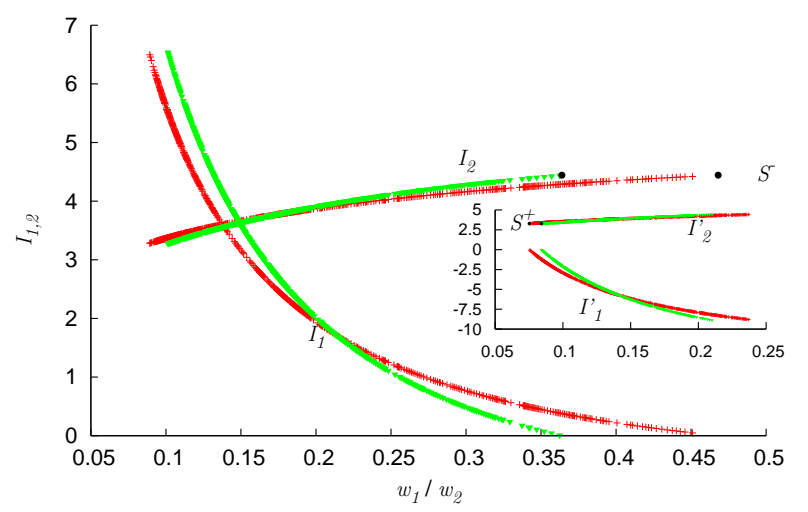

FIG. 4: (color online). Action variables for the $T_{2}^{\mathrm{p}}$ (red plus symbols) and the $T_{2}^{\mathrm{n}}$ (green triangles) families at $E=$ -1.5 a.u., $F=0.5$ a.u. Black dots show the action and the stability angles of the organizing center $S^{-}$. The inset presents the same situation in a different action-angle coordinate system, in which $S^{+}$can be identified as another organizing center of the $T_{2}^{\mathrm{p}, \mathrm{n}}$ families.

identify the angles conjugate to $I_{2}$ and $I_{1}$ as the degrees of freedom along and transverse to the central FPO $S^{-}$.

At the lower limits of the winding ratios in Fig. [4 none of the action variables converges to zero, and it seems, therefore, that, contrary to the hypothesis derived from Fig. 11 the $T_{2}^{\mathrm{p}}$ and $T_{2}^{\mathrm{n}}$ families are not organized by $S^{+}$ the way they are by $S^{-}$. However, this difference is an artifact of the action-angle coordinate system. In fact, Fourier spectra suggest that for 2-torus POs close to $S^{+}$ a set of winding numbers $\mathbf{w}^{\prime}$ should be used that starts the counting scheme with $w_{2}=4$ for the shortest orbit in the leftmost series. This set of winding numbers leads to the action variables $\mathbf{I}^{\prime}$ used in the inset of Fig. 4 In this coordinate system, the approach to $S^{+}$satisfies the three criteria set up above, whereas the approach to $S^{-}$ does not. Therefore, both FPOs $S^{+}$and $S^{-}$serve as organizing centers for the two families of 2-tori POs in an equal manner. (The POs in Fig. 1 1 are thus labeled in the coordinate system induced by the nearest FPO.)

For the $T_{2}^{\mathrm{p}}$, the conclusion that $S^{+}$and $S^{-}$serve as organizing centers for families of 2-torus POs has already been reached from the Poincaré surface of section plot in Fig. 2. Notice that in spite of its intuitive appeal the Poincaré plot is somewhat misleading because it seems to show two distinct families of 2-torus POs. In fact, however, the $T$-S-plot of Fig. 1 indicates, and the assignment of winding numbers confirms, that all planar 2torus POs form the single family $T_{2}^{\mathrm{p}}$. A two-dimensional Poincaré surface of section is entirely unsuitable to analyze the geometry of the $T_{2}^{\mathrm{n}}$ because these POs do not lie in a two-dimensional plane. Nevertheless, the quantitative method developed here demonstrates that the relation of $T_{2}^{\mathrm{n}}$ to the FPOs $S^{ \pm}$is the same as that of the $T_{2}^{\mathrm{p}}$. Apart from avoiding this ambiguity, our method for the analysis of phase space topology is also more general 

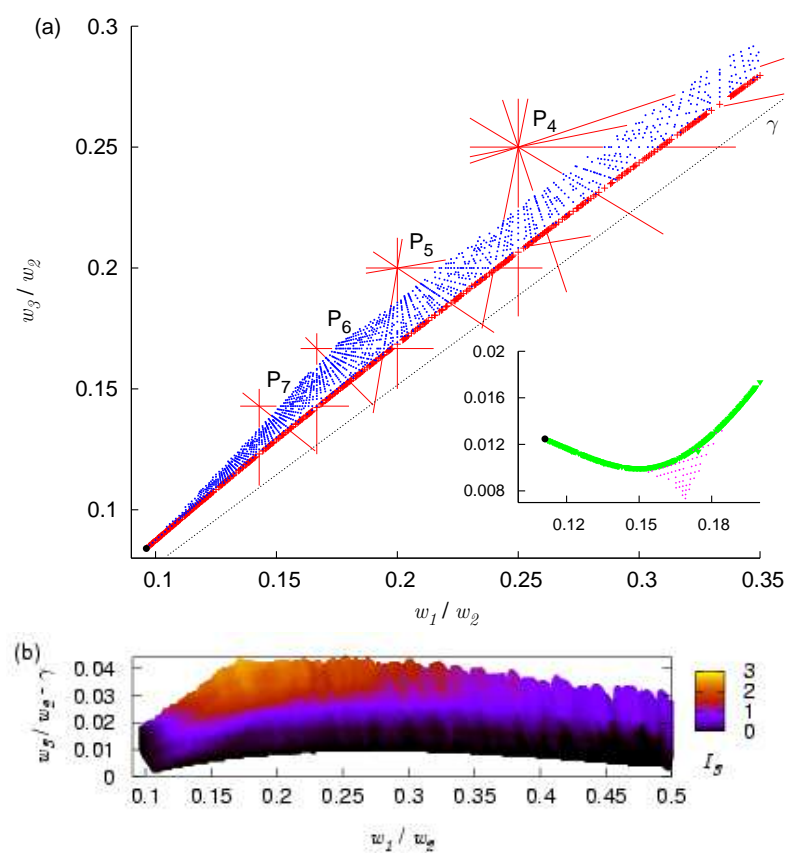

FIG. 5: (color online). (a) Winding ratios for POs from the $T^{\mathrm{p}}$ families at $E=-1.4$ a.u., $F=0.5$ a.u. with the same symbols as in Fig. 11 The most prominent resonance lines [7, 17, 18] are indicated. The inset shows POs from the $T^{\mathrm{n}}$ families. (b) Action variable $I_{3}$ for the $T_{3}^{\mathrm{p}}$ family. For clarity the dotted line $\gamma$ from (a) has been subtracted on the vertical axis.

than Poincaré surface of section techniques in that it can now readily be applied to the 3 -torus POs.

The 3-torus POs in Fig. 1 fall into the same series as the 2-torus POs, but they possess a third winding number $w_{3}$. The latter manifests itself in an additional major peak in the Fourier spectra of the coordinates, and therefore can be assigned by a straightforward extension of the technique used to classify the 2-torus POs.

Fig. 5 (a) shows the winding ratios of 2 - and 3 -torus POs. For the 2 -torus POs, the missing ratio $w_{3} / w_{2}$ is replaced with the stability angle that describes the dynamics transverse to the original torus. The stability angles of the 2-torus POs limit the winding ratios of the 3-torus POs. As this limit is approached, the action variable $I_{3}$

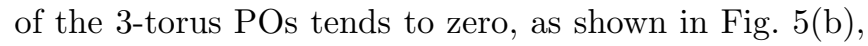
whereas $I_{1}$ and $I_{2}$ converge toward the values obtained from the 2-torus POs. These three observations are entirely analogous to the three criteria laid out above that characterize the relation of a family of 2 -torus POs to its organizing FPOs. We can therefore conclude that we are here presented with a higher-dimensional generalization of that situation: The $T_{2}$ families serve as organizing centers for the 3 -torus POs, and they impose a distinguished coordinate system onto the $T_{3}$ in which $w_{3}$ and the action $I_{3}$ identify the direction transverse to the $T_{2}$.

In summary, we have demonstrated how periodic or- bits in a non-integrable multidimensional Hamiltonian system can be used to reconstruct a hierarchy of phase space structures that is organized by a stable $\mathrm{PO}$ at its center. The POs on each level of this hierarchy serve as organizing centers for the POs of the next-higher level. We have derived three general criteria to diagnose this relation between POs. They are independent of the underlying dynamical system and can therefore be expected to be of wide applicability. At the same time, the calculation of the associated action variables provides the key prerequisite for an Einstein-Brillouin-Keller torus quantization that the crossed-fields hydrogen atom has so far resisted. It thereby paves the way to an immediate, and important, semiclassical application of the purely classical results obtained here. These results will be presented in a forthcoming publication.

We thank C. Chandre, À. Jorba, J. D. Meiss, and G. Wunner for helpful comments and remarks. This work was supported by the Deutsche Forschungsgemeinschaft, Deutscher Akademischer Austauschdienst, National Science Foundation, and the Alexander von HumboldtFoundation.

[1] H. Poincaré, Les méthodes nouvelles de la mécanique céleste (Imprimerie Gauthier-Villars et fils, Paris, 1892), vol. 1, chap. III, p. 82, [authors' translation].

[2] V. Szebehely, Theory of orbits (Academic Press, 1967).

[3] F. Gabern and À. Jorba, Discrete Contin. Dynam. Systems Series B 1, 143 (2001).

[4] R. Prosmiti and S. C. Farantos, J. Chem. Phys. 118, 8275 (2003).

[5] M. Joyeux and L. Michaille, ACH - Models in Chemistry 134, 573 (1997).

[6] O. Hahn, J. M. G. Llorente, and H. S. Taylor, J. Chem. Phys. 94, 2608 (1991).

[7] D. Robin, C. Steier, J. Laskar, and L. Nadolski, Phys. Rev. Lett. 85, 558 (2000).

[8] M. C. Gutzwiller, Chaos in Classical and Quantum Mechanics (Springer, New York, 1990).

[9] G. Raithel, M. Fauth, and H. Walther, Phys. Rev. A 44, 1898 (1991).

[10] D. M. Wang and J. B. Delos, Phys. Rev. A 63, 043409 (2001).

[11] G. Kawahara and S. Kida, J. Fluid. Mech. 449, 291 (2001).

[12] G. Stania and H. Walther, Phys. Rev. Lett. 95, 194101 (2005).

[13] E. Flöthmann, J. Main, and K. H. Welge, J. Phys. B 27, 2821 (1994).

[14] E. Flöthmann and K. H. Welge, Phys. Rev. A 54, 1884 (1996).

[15] V. I. Arnold, Mathematical Methods of Classical Mechanics (Springer, 1989).

[16] H. Kook and J. D. Meiss, Physica D 35, 65 (1989).

[17] J. Laskar, Physica D 67, 257 (1993).

[18] J. von Milczewski, G. H. F. Diercksen, and T. Uzer, Phys. Rev. Lett. 76, 2890 (1996). 